\title{
Involvement of activated prorenin in the pathogenesis of slowly progressive nephropathy in the non-clipped kidney of two kidney, one-clip hypertension
}

\author{
Masaki Ryuzaki ${ }^{1}$, Atsuhiro Ichihara ${ }^{1,2}$, Yoichi Ohshima ${ }^{1}$, Mariyo Sakoda ${ }^{2}$, Asako Kurauchi-Mito ${ }^{2}$, \\ Tatsuya Narita ${ }^{2}$, Kenichiro Kinouchi ${ }^{2}$, Kanako Murohashi-Bokuda ${ }^{2}$, Akira Nishiyama ${ }^{3}$ and Hiroshi Itoh ${ }^{2}$
}

The handle region peptide (HRP), a (pro)renin receptor (P)RR blocker, did not prevent the acute nephropathy occurring 2 weeks after clipping in renovascular hypertensive rats. This study was performed to examine the effects of HRP, its scramble peptide, or a saline vehicle on slowly progressive nephropathy occurring in the kidneys of two-kidney, one-clip Goldblatt hypertensive rats. At 2 weeks after clipping, the renal morphology in the clipped and non-clipped kidneys was similar in the three groups of rats. At 12 weeks after clipping, however, the glomerulosclerosis index (GI) and the tubulointerstitial damage (TD) of the non-clipped kidneys of the HRP-treated rats were significantly lower than those of vehicle-treated rats, although the GI and the TD were similar in the rats treated with scramble peptide and vehicle. The GI and the TD of the clipped kidneys were similar in the three groups of rats at 12 weeks after clipping. In the non-clipped kidneys at 12 weeks after clipping, activated prorenin levels, angiotensin II levels and transforming growth factor (TGF)- $\beta$ mRNA levels of HRP-treated rats were significantly lower than those of vehicle-treated rats, although they were similar in the non-clipped kidneys from the rats treated with scramble peptide and vehicle. In the clipped kidneys at 12 weeks after clipping, activated prorenin levels, angiotensin II levels and TGF- $\beta$ mRNA levels were similar in the three groups of rats. These results suggest that the ((P)RR)-dependent activation of prorenin contributes to the pathogenesis of slowly progressive nephropathy in the intact kidney in a rat model of renovascular hypertension.

Hypertension Research (2011) 34, 301-307; doi:10.1038/hr.2010.230; published online 25 November 2010

Keywords: angiotensin; handle region peptide; (pro)renin receptor; renovascular hypertension; transforming growth factor- $\beta$

\section{INTRODUCTION}

The (pro)renin receptor $(\mathrm{P}) \mathrm{RR}$ is found in the human kidney, and its binding to prorenin causes the non-proteolytic activation of prorenin, leading to the generation of tissue angiotensin (Ang) peptides. ${ }^{1}$ However, the physiological functions or the pathophysiological roles of the $(\mathrm{P}) \mathrm{RR}$, remains unknown. Recent studies have clearly shown that synthetic peptides containing the amino acid sequence corresponding to the handle region of prorenin bind to the $(\mathrm{P}) \mathrm{RR}$ and inhibit the binding of prorenin to the receptor. ${ }^{2,3}$ Thus, the (P)RR clearly binds to the handle region of prorenin, and handle region peptide (HRP) is thought to be a potent (P)RR blocker. The in vivo administration of HRP $\left(3.5 \mu \mathrm{g}-1.0 \mathrm{mg} \mathrm{kg}^{-1}\right.$ per day) for $8-24$ weeks showed beneficial effects on the slowly progressive nephropathy developed in streptozotocin-induced type I diabetic rats, ${ }^{4}$ type II diabetic $\mathrm{db} / \mathrm{db}$ mice, ${ }^{5}$ spontaneously hypertensive rats, ${ }^{6}$ and strokeprone spontaneously hypertensive rats. ${ }^{7}$ In contrast, the same dose of HRP did not inhibit the kidney damage that occurred at 2 weeks after clipping in two-kidney, one-clip (2K1C) renovascular hypertensive rats. ${ }^{8}$ Generally, the damage in the clipped kidney occurs acutely after clipping, and the damage in the non-clipped kidney starts appearing approximately 2 weeks after clipping. Thus, the (P)RR may contribute to the pathogenesis of slow, non-acute organ damage.

In the present study, we hypothesized that (P)RR-dependent mechanisms are involved in slowly progressive nephropathy, but not in acutely developed nephropathy, in 2K1C renovascular hypertension. To test this hypothesis, the effects of treatment with HRP on the nephropathy occurring at 2 and 12 weeks after clipping in the clipped and non-clipped kidneys of $2 \mathrm{~K} 1 \mathrm{C}$ renovascular hypertensive rats were examined.

\section{METHODS}

Animals

Male Sprague-Dawley rats (SD; Sankyo Laboratory, Tokyo, Japan) were maintained in a temperature-controlled room at $23^{\circ} \mathrm{C}$ with a 12:12-h light-dark cycle. The rats were given unlimited access to water and rat chow (CE-2; Nihon Clea, Tokyo, Japan). At 4 weeks of age, 2K1C renovascular

\footnotetext{
${ }^{1}$ Department of Endocrinology and Anti-Aging Medicine, Keio University School of Medicine, Tokyo, Japan; ${ }^{2}$ Department of Internal Medicine, Keio University School of Medicine, Tokyo, Japan and ${ }^{3}$ Department of Pharmacology, Kagawa University School of Medicine, Kagawa, Japan Correspondence: Dr A Ichihara, Endocrinology and Anti-Aging Medicine, Keio University School of Medicine, 35 Shinanomachi, Shinjuku-ku, Tokyo 160-8582, Japan. E-mail: atzichi@sc.itc.keio.ac.jp
}

Received 28 June 2010; revised 2 August 2010; accepted 23 August 2010; published online 25 November 2010 
hypertension was induced in all rats by placing a titanium clip of $0.2-\mathrm{mm}$ ID around the left renal artery. Additionally, we implanted a telemetry transmitter probe (model TA11PA-C40; Data Sciences International, St Paul, MN, USA) into the rats. The flexible tip of the probe was positioned immediately below the renal arteries. The transmitter was then surgically sutured into the abdominal wall, and the incision was closed. Furthermore, an osmotic minipump (model 2004 for 28-day use; Alzet, Cupertino, CA, USA) containing a (P)RR blocker, HRP (HRP: NH2-RILLKKMPSV-COOH) or its scramble peptide (NH2-KSIRVLPKML-COOH) was subcutaneously implanted in each rat. Osmotic minipumps were replaced every 4 weeks. The rats were divided into three groups: rats treated with vehicle as control, rats treated with $1.0 \mathrm{mg} \mathrm{kg}^{-1}$ per day of HRP and rats treated with $1.0 \mathrm{mg} \mathrm{kg}^{-1}$ per day of scramble peptide. As shown in our previous study, ${ }^{9}$ treatment with $1.0 \mathrm{mg} \mathrm{kg}^{-1}$ per day of HRP achieved serum HRP levels averaging $110 \mathrm{~nm}$, a dose reportedly large enough to inhibit prorenin binding to the (P)RR. ${ }^{2}$ After all the procedures were performed, rats were returned to their home cages and allowed to recover for 4 days before starting any measurements. All of the procedures were performed, whereas the rats were under sodium pentobarbital anesthesia $\left(50 \mathrm{mg} \mathrm{kg}^{-1}\right.$, i.p.). The Keio University animal care and use committee approved all of the experimental protocols, including the placement of titanium clips and the implantation of both minipumps and radiotelemetry transmitters.

\section{Experimental protocols}

Body weight was monitored once every week. We monitored the mean arterial pressure in unrestricted, untethered and conscious animals using the Dataquest IV system (Data Sciences International), which consists of an implanted radiofrequency transmitter and a receiver placed under each cage. The output was relayed from the receiver through a consolidation matrix to a personal computer. Individual 10-s mean arterial pressures were sampled every 5 min for $24 \mathrm{~h}$ on the days that represented exactly 2 and 12 weeks after clipping; average and s.d. were calculated. At 2 and 12 weeks after clipping, a 24-h urine sample was collected using a metabolic cage, and the urinary protein excretion level was determined with a Micro TP test kit (Wako, Osaka, Japan). After measuring the arterial pressure and sampling the urine, the rats were decapitated to collect their blood and kidneys. Using the collected blood, plasma renin activity was determined by a radioimmunoassay (Dinabott Radioisotope Institute, Tokyo, Japan).

\section{Morphological evaluation}

The cortex parts of the clipped and the non-clipped kidneys removed from each rat were fixed in $4 \%$ paraformaldehyde in phosphate buffer ( $\mathrm{pH} 7.4$ ); paraffin-embedded sections of both the clipped and the non-clipped kidneys were stained using the Periodic acid Schiff method, and renal-activated prorenin was assessed by immunostaining for the 'gate region' of prorenin. ${ }^{7}$ For immunohistochemical staining of non-proteolytically activated prorenin, the anti-rat gate region antibody was applied to the sections as the primary antibody. The sections were incubated with a biotin-conjugated anti-rabbit IgG as the secondary antibody, and the antibody reactions were visualized with a Vectastain ABC standard Kit (Vector Laboratories, Burlingame, CA, USA) and an AEC Standard Kit (Dako, Glostrup, Denmark) following the manufacturer's instructions. We quantitatively determined the immunoreactive nonproteolytically activated prorenin-positive area in each glomerulus at $\times 200$ magnification with a Mac SCOPE (Version 2.5; Mitani, Fukui, Japan) and expressed it as a percentage of the whole cross-sectional area of the glomerulus. ${ }^{7}$

The glomerulosclerosis index (GI) of each sample was determined in a double-blind manner by Nihon Biological Laboratories (Tokyo, Japan). A GI was derived for each animal by examining 100 glomeruli at $\times 400$ magnification. The severity of glomerulosclerosis was expressed on an arbitrary scale from 0 to 4: grade 0 , normal glomeruli; grade 1, presence of mesangial expansion/thickening of the basement membrane; grade 2, mild to moderate segmental hyalinosis/sclerosis, involving less than $50 \%$ of the glomerular tuft; grade 3, diffuse glomerular hyalinosis/sclerosis, involving more than $50 \%$ of the tuft; grade 4, diffuse glomerulosclerosis, with total tuft obliteration and collapse as shown previously. ${ }^{4}$ Tubular (atrophy, casts and dilation) and interstitial (fibrosis and inflammation) changes were graded on a scale of
0 to $4+(0$, no changes; $1+$, changes affecting $<25 \%$ of the sample; $2+$, changes affecting $25-50 \%$ of the sample; $3+$, changes affecting $50-75 \%$ of the sample; $4+$, changes affecting $75-100 \%$ of the sample). ${ }^{10}$ The resulting index in each animal was expressed as a mean of all scores obtained.

\section{Measurements of angiotensin peptides}

The cortex regions of the removed kidneys were weighed and placed in ice-cold methanol $(10 \% \mathrm{wt} / \mathrm{vol})$, homogenized with a chilled glass homogenizer and centrifuged. The supernatant was then dried and reconstituted in $4 \mathrm{ml}$ of $50 \mathrm{~mm}$ sodium phosphate buffer containing $1 \%$ albumin. Reconstituted samples were extracted using a Bond-Elut column (Analytichem, Harbor City, CA, USA), and the eluents were evaporated to dryness and reconstituted in angiotensin peptide assay diluent. The angiotensin II content was quantitatively determined using a radioimmunoassay with rabbit anti-angiotensin II antiserum (Arnel, New York, NY, USA) as shown previously, ${ }^{4}$ which followed the methods of Dr Navar's laboratory (New Orleans, LA, USA). ${ }^{11}$ The standard curve of the AngII radioimmunoassay was from 0.39 to $400 \mathrm{fmol} \mathrm{ml}^{-1}$. We checked the intra- and interassay variabilities using $20 \mathrm{fmol} \mathrm{ml}^{-1}$ of an internal standard and by measuring the same samples for different assays.

\section{Real-time quantitative RT-PCR analysis}

In this experiment, the RNeasy Mini kit (Qiagen, Tokyo, Japan) was used to extract the total RNA from the cortex regions of the kidney removed from each animal. Then, real-time quantitative RT-PCR using the TaqMan One-Step RT-PCR Master Mix Reagents Kit (Applied Biosystems, Foster City, CA, USA) and an ABI Prism 7700 HT Detection System (Applied Biosystems, Carlsbad, CA, USA) was performed. The probes and primers used for the rat genes encoding the angiotensin-converting enzyme (ACE), angiotensinogen, renin, (P)RR, transforming growth factor-beta (TGF- $\beta$ ) and glyceraldehyde-3-phosphate dehydrogenase have been previously described. ${ }^{12,13}$

\section{Statistical analyses}

The within-group statistical comparisons were made using a one-way ANOVA for repeated measures followed by the Newman-Keuls post hoc test. Differences between groups were evaluated using a two-way ANOVA for repeated measures combined with the Newman-Keuls post hoc test. $P<0.05$ was considered significant. Data are reported as the mean \pm standard deviation (s.d.). Calculations were carried out using Statview 5.0 software (SAS, Cary, NC, USA).

\section{RESULTS}

Body weight, mean arterial pressure, urinary protein excretion and plasma renin activity

As shown in Figure 1, the body weight of the rats treated with vehicle $(n=23)$, HRP $(n=23)$ and scramble peptide $(n=6)$ increased during the 12-week observation period, and their increases were similar among the three groups.

Neither treatment with HRP nor with scramble peptide significantly altered mean arterial pressure at 2 or 12 weeks after clipping. Mean arterial pressures at 2 and 12 weeks after clipping averaged $100 \pm 10 \mathrm{~mm} \mathrm{Hg}(n=4)$ and $180 \pm 10 \mathrm{~mm} \mathrm{Hg}(n=4)$, respectively, in vehicle-treated rats; $105 \pm 6 \mathrm{~mm} \mathrm{Hg}(n=4)$ and $175 \pm 12 \mathrm{~mm} \mathrm{Hg}(n=6)$, respectively in HRP-treated rats; and $100 \pm 10 \mathrm{~mm} \mathrm{Hg}(n=4)$ and $175 \pm 8 \mathrm{~mm} \mathrm{Hg}(n=4)$, respectively, in scramble peptide-treated rats.

Similar results were observed for the urinary protein excretion. Urinary protein excretion at 2 and 12 weeks after clipping was not different among the three groups and averaged $16.3 \pm 10.2 \mathrm{mg}$ per day $(n=8)$ and $43.3 \pm 46.6 \mathrm{mg}$ per day $(n=13)$, respectively, in vehicletreated rats; $21.6 \pm 15.3 \mathrm{mg}$ per day $(n=8)$ and $83.0 \pm 102.2 \mathrm{mg}$ per day $(n=14)$, respectively, in HRP-treated rats; and $15.9 \pm 11.8 \mathrm{mg}$ per day $(n=6)$ and $91.2 \pm 91.5 \mathrm{mg}$ per day $(n=6)$, respectively, in scramble peptide-treated rats. There was no significant difference in plasma renin activity between the two time points of 2 and 12 weeks after clipping or among the three treatment groups. 
a

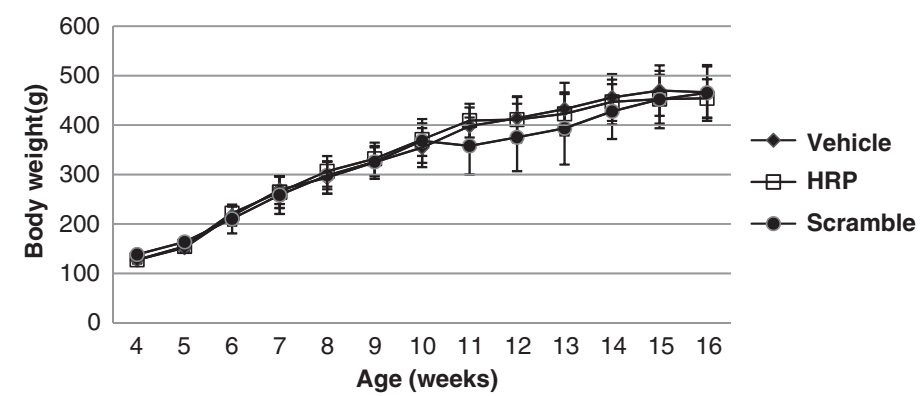

b

6 weeks of age (2 weeks after clipping)

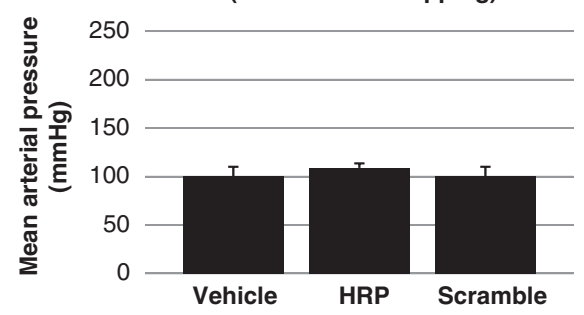

C

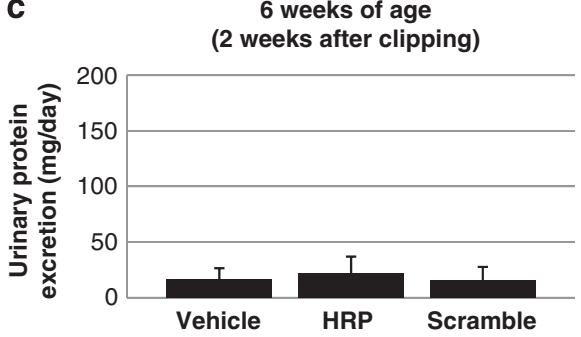

d

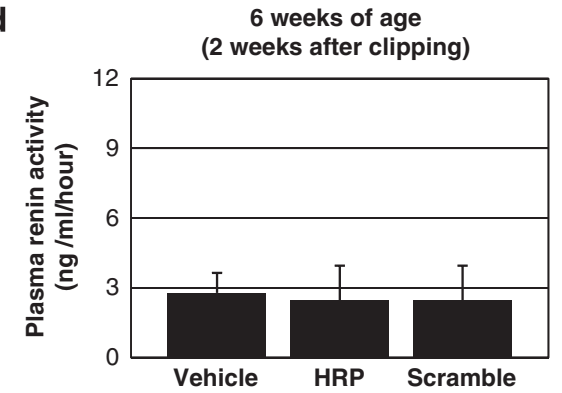

16 weeks of age (12 weeks after clipping)
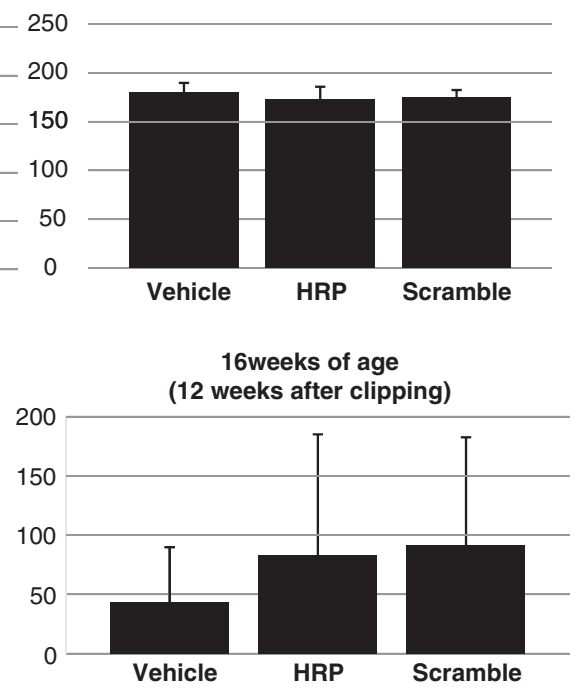

16 weeks of age

16 weeks of age (12 weeks after clipping)

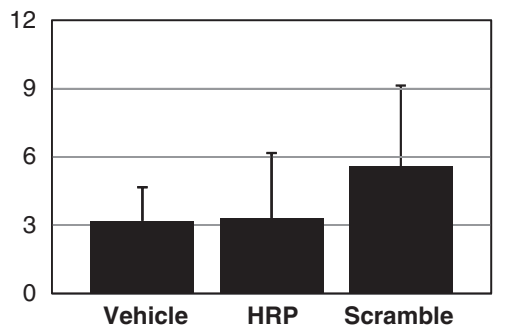

Figure 1 (a) Body weight during the observation period in two-kidney, one-clip Goldblatt hypertensive rats treated with vehicle, HRP and scramble peptide. (b) Mean arterial pressure during the observation period in two-kidney, one-clip Goldblatt hypertensive rats treated with vehicle, HRP and scramble peptide. (c) Urinary protein excretion during the observation period in two-kidney, one-clip Goldblatt hypertensive rats treated with vehicle, HRP and scramble peptide. (d) Plasma renin activity in two-kidney, one-clip Goldblatt hypertensive rats treated with vehicle, HRP and scramble peptide.

\section{Renal morphology}

At 2 weeks after clipping, renal damage was minimal, in both the clipped and the non-clipped kidneys, as expected. The renal morphology, GI and tubulointerstitial damage (TD) index were similar in the rats treated with vehicle, HRP and scramble peptide (Figure 2).

At 12 weeks after clipping, however, diffuse glomerulosclerosis and TD were observed in the non-clipped kidneys of the rats treated with vehicle and scramble peptide, whereas only a few glomerulosclerosis was observed in the non-clipped kidneys of the rats treated with HRP (Figure 2b, lower). The GI of the non-clipped kidneys of HRP-treated rats $(0.31 \pm 0.04, n=9)$ was significantly lower than that of rats treated with vehicle $(0.72 \pm 0.15, n=8)$, whereas the GI of scramble peptidetreated rats $(0.70 \pm 0.13, n=3)$ was similar to that of rats treated with vehicle (Figure 2c, right). At 12 weeks after clipping, the TD index of non-clipped kidneys of HRP-treated rats $(0.6 \pm 0.5, n=9)$ was significantly lower than that of vehicle-treated rats $(2.3 \pm 0.5, n=8)$, whereas the TD index of scramble peptide-treated rats $(1.7 \pm 0.6, n=3)$ was similar to that of vehicle-treated rats (Figures 2b, lower and Figure 2d, right).

In the clipped kidneys, at 12 weeks after clipping, the renal morphology and GI were similar in the three groups (Figure $2 b$, upper and Figure 2c, left). The TD was less with HRP treatment. However, the index of the rats treated with vehicle, HRP and scramble peptide averaged $1.6 \pm 0.5(n=8), 0.7 \pm 0.5(n=9)$ and $1.7 \pm 0.6(n=3)$, respectively, and was not statistically different among the three groups (Figure 2b, upper and Figure 2d, left). 

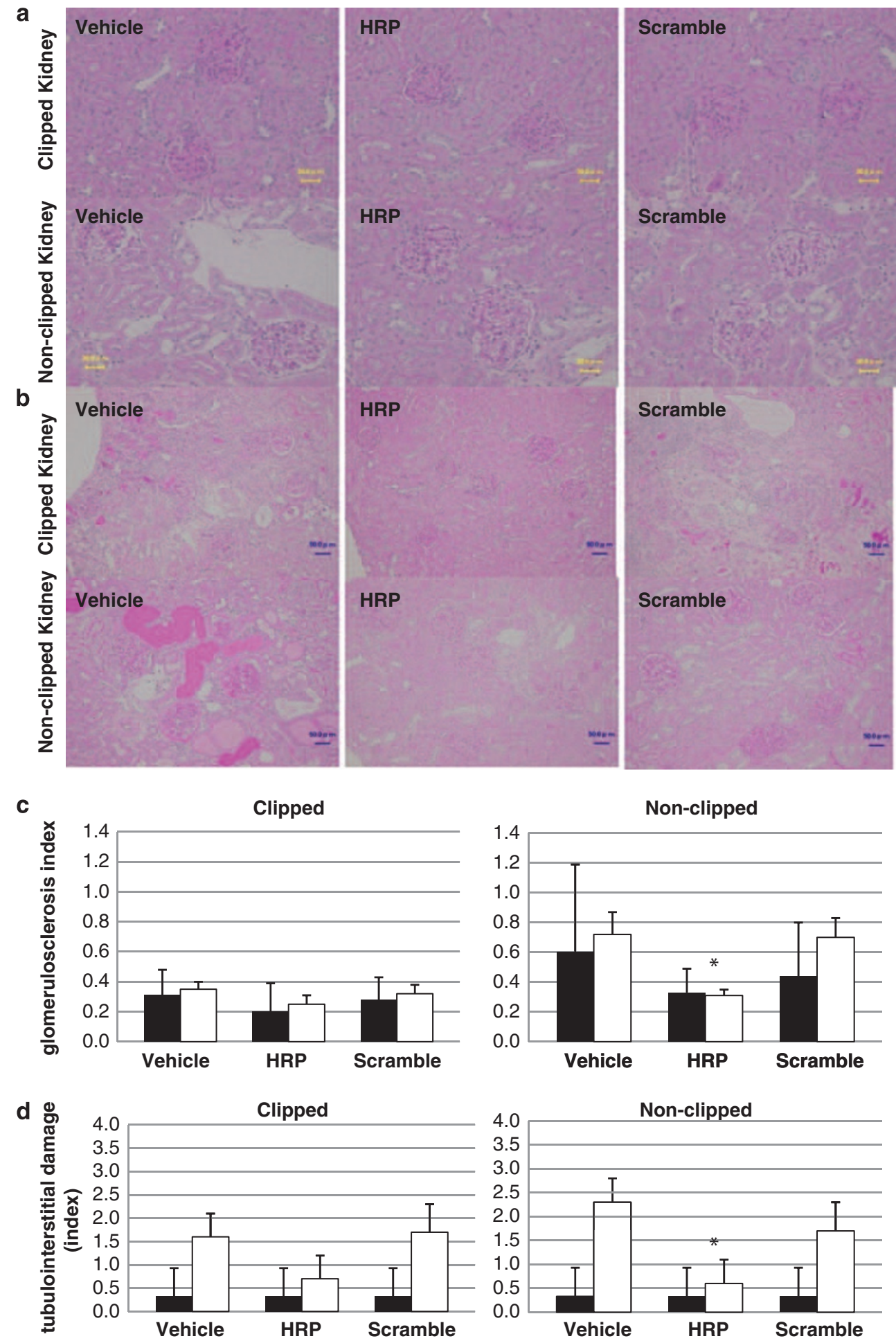

Figure 2 Representative renal morphology at 2 (a, bar scale; $30 \mu \mathrm{m})$ and 12 (b, bar scale; $50 \mu \mathrm{m}$ ) weeks after clipping, glomerulosclerosis index at 2 (closed bar) and 12 (open bar) weeks after clipping (c), and tubulointerstitial damage at 2 (closed bar) and 12 (open bar) weeks after clipping (d) of the clipped and the non-clipped kidneys of two-kidney, one-clip Goldblatt hypertensive rats treated with vehicle ( $n=3$ at 2 weeks and $n=8$ at 12 weeks after clipping), HRP ( $n=3$ at 2 weeks and $n=9$ at 12 weeks after clipping) and scramble peptide ( $n=3$ at 2 weeks and $n=3$ at 12 weeks after clipping). ${ }^{*} P<0.05$ vs. vehicle.

\section{Renal angiotensin II level}

As shown in Figure 3, in both the clipped kidneys and the non-clipped kidneys at 2 weeks after clipping, there was no significant difference among the three groups of rats. At 12 weeks after clipping, however, renal Ang II levels in the non-clipped kidneys of the rats treated with HRP were significantly lower than that in rats treated with vehicle, whereas the levels in the scramble peptide-treated rats were similar to that of rats treated with vehicle. In the clipped kidneys, at 12 weeks after clipping, angiotensin II levels were similar in the three groups. 


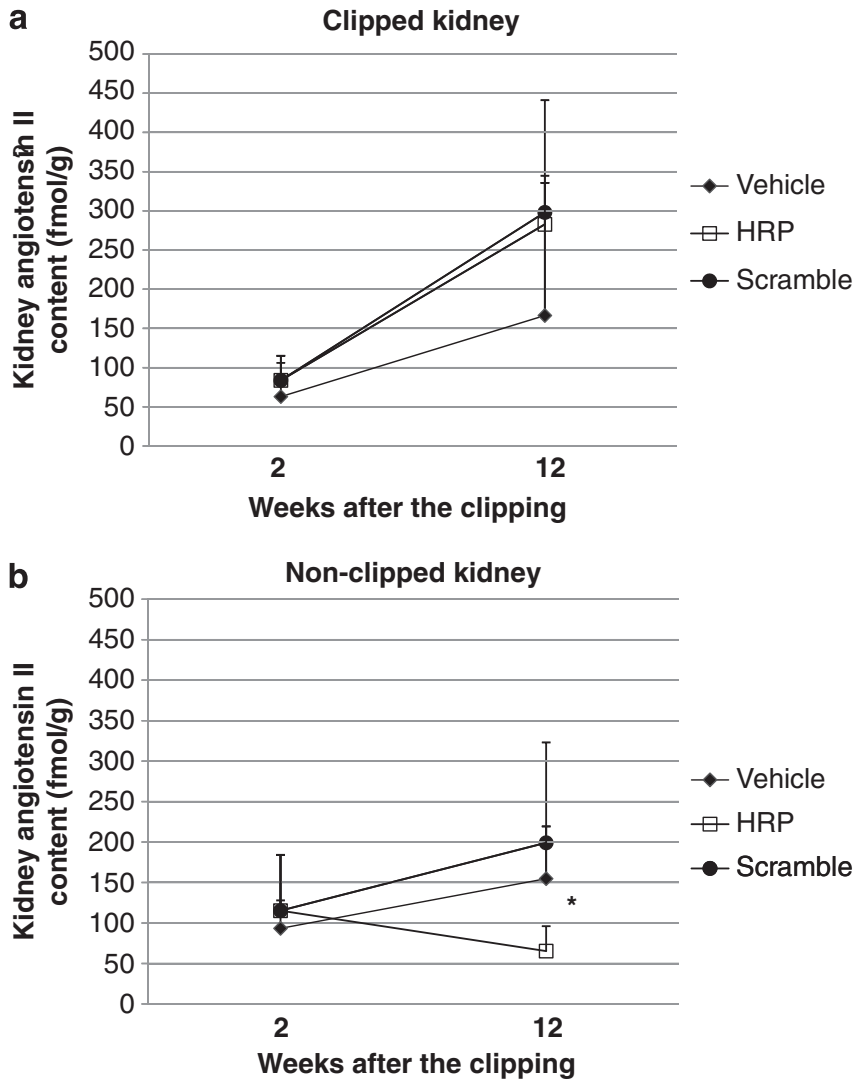

Figure 3 Renal angiotensin II levels at 2 and 12 weeks after clipping in the clipped (a) and the non-clipped kidneys (b) of two-kidney, one-clip Goldblatt hypertensive rats treated with vehicle ( $n=6$ and 8 , respectively), HRP ( $n=6$ and 8 , respectively) and scramble peptide ( $n=6$ and 3 , respectively). ${ }^{*} P<0.05$ vs. vehicle.

\section{Renal-activated prorenin}

At 2 weeks after clipping, immunostaining for activated prorenin were similar among the three groups in both the clipped kidneys and the non-clipped kidneys (Figure 4).

At 12 weeks after clipping, strong immunostaining for activated prorenin was observed in the non-clipped kidneys of untreated rats and scramble peptide-treated rats. However, only a weak immunostaining for activated prorenin was observed in the non-clipped kidneys of HRP-treated rats (Figure 5). In the clipped kidneys, the immunostaining for activated prorenin was similar in the three groups (Figure 5).

\section{Renal mRNA levels of the renin-angiotensin components and TGF- $\beta$}

As shown in Table 1, renal mRNA levels of angiotensinogen, renin and (P)RR were similar in the three groups in both the clipped kidneys and the non-clipped kidneys at 2 and 12 weeks after clipping.

In the clipped kidneys of rats taken at 12 weeks after clipping, the renal ACE mRNA levels of the rats treated with HRP and scramble peptide were significantly lower than that of rats treated with vehicle. In both the clipped and the non-clipped kidneys at 2 weeks after clipping, and in the non-clipped kidneys at 12 weeks after clipping, ACE mRNA levels were similar in the three groups.

In the non-clipped kidneys of rats taken at 12 weeks after clipping, TGF- $\beta$ mRNA levels of HRP-treated rats were significantly lower than that of untreated rats. However, the TGF- $\beta$ mRNA levels of scramble
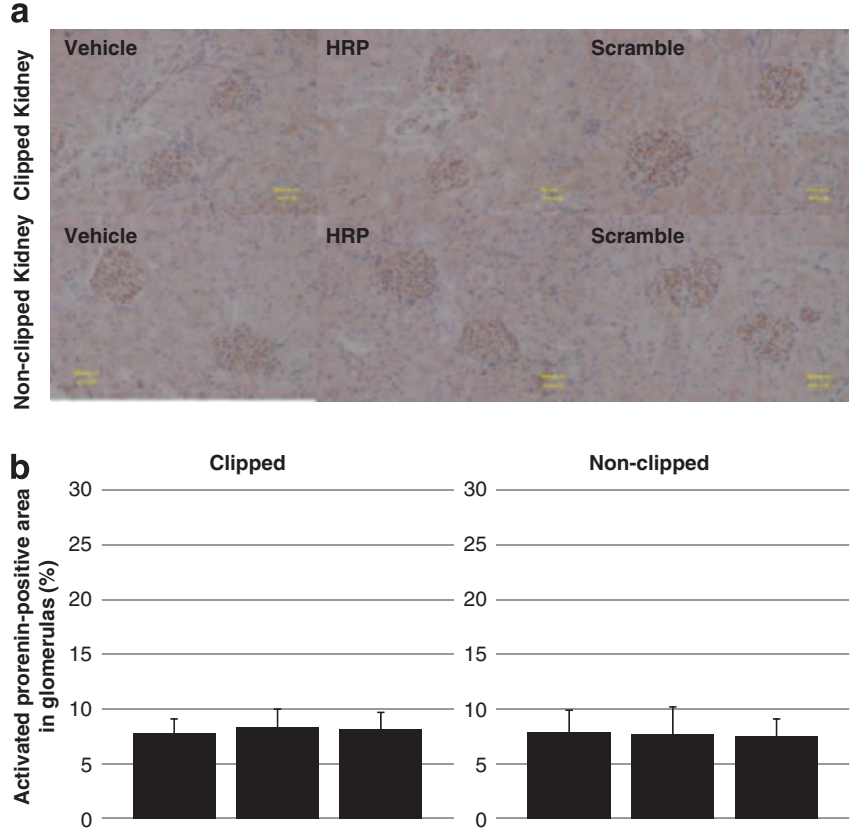

Figure 4 Representative immunostaining for the 'gate region' of prorenin (a) and the quantitative assessment of renal-activated prorenin (b) at 2 weeks after clipping in two-kidney, one-clip Goldblatt hypertensive rats treated with vehicle $(n=3), \operatorname{HRP}(n=3)$ and scramble peptide $(n=3)$.

a
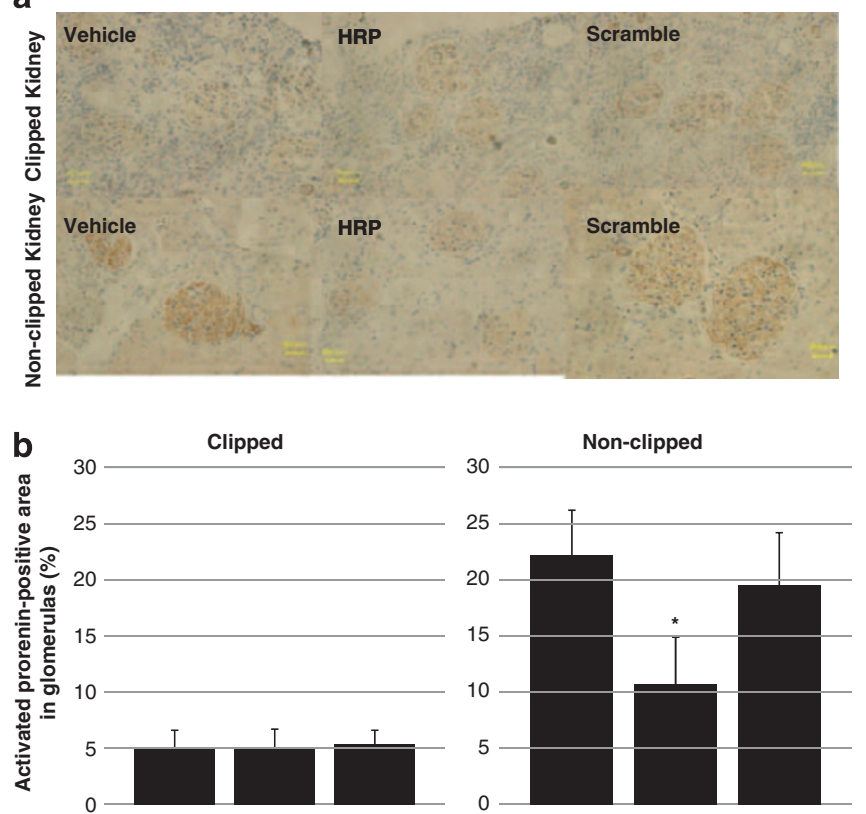

Figure 5 Representative immunostaining for the 'gate region' of prorenin (a) and the quantitative assessment of renal-activated prorenin (b) at 12 weeks after clipping in two-kidneys, one-clip Goldblatt hypertensive rats treated with vehicle $(n=8)$, HRP $(n=9)$ and scramble peptide $(n=3)$. ${ }^{*} P<0.05$ vs. vehicle.

peptide-treated rats were similar to that of untreated rats. In both the clipped and the non-clipped kidneys at 2 weeks after clipping, and in clipped kidneys at 12 weeks after clipping, TGF- $\beta$ mRNA levels were similar in the three groups. 
Table 1 Renal mRNA levels (ratio to GAPDH mRNA) of the renin-angiotensin system components and TGF- $\beta$ in two-kidney, one-clip Goldblatt hypertensive rats

6 weeks of age (2 weeks after clipping)

\begin{tabular}{|c|c|c|c|c|c|c|}
\hline \multirow[b]{2}{*}{ Treatment } & \multicolumn{3}{|c|}{ Clipped kidney } & \multicolumn{3}{|c|}{ Non-clipped kidney } \\
\hline & Vehicle $(\mathrm{N}=6)$ & $\operatorname{HRP}(\mathrm{N}=6)$ & Scramble $(\mathrm{N}=6)$ & Vehicle $(\mathrm{N}=6)$ & $\operatorname{HRP}(\mathrm{N}=6)$ & Scramble $(\mathrm{N}=6)$ \\
\hline ACE & $2.0 \pm 1.5$ & $2.3 \pm 0.9$ & $2.1 \pm 1.1$ & $0.8 \pm 0.3$ & $0.9 \pm 0.3$ & $1.0 \pm 0.4$ \\
\hline AGT & $0.6 \pm 0.3$ & $1.5 \pm 1.3$ & $1.4 \pm 0.9$ & $0.6 \pm 0.9$ & $0.9 \pm 0.6$ & $0.9 \pm 0.5$ \\
\hline Rennin & $18.3 \pm 15.0$ & $17.5 \pm 17.5$ & $17.4 \pm 14.0$ & $7.1 \pm 9.8$ & $6.6 \pm 10.1$ & $6.4 \pm 9.8$ \\
\hline (P)RR & $1.0 \pm 0.9$ & $1.3 \pm 0.5$ & $1.2 \pm 0.4$ & $0.9 \pm 0.2$ & $0.8 \pm 0.1$ & $0.9 \pm 0.2$ \\
\hline TGF- $\beta$ & $1.5 \pm 2.6$ & $1.6 \pm 2.2$ & $1.5 \pm 2.0$ & $1.3 \pm 0.3$ & $1.1 \pm 0.1$ & $1.2 \pm 0.2$ \\
\hline
\end{tabular}

16 weeks of age (12 weeks after clipping)

\begin{tabular}{|c|c|c|c|c|c|c|}
\hline \multirow[b]{2}{*}{ Treatment } & \multicolumn{3}{|c|}{ Clipped kidney } & \multicolumn{3}{|c|}{ Non-clipped kidney } \\
\hline & Vehicle $(\mathrm{N}=7)$ & $\operatorname{HRP}(\mathrm{N}=6)$ & Scramble $(\mathrm{N}=3)$ & Vehicle $(\mathrm{N}=7)$ & $\operatorname{HRP}(\mathrm{N}=6)$ & Scramble $(\mathrm{N}=3)$ \\
\hline ACE & $3.3 \pm 1.1$ & $1.9 \pm 0.9 *$ & $1.2 \pm 0.4^{*}$ & $2.0 \pm 0.6$ & $2.1 \pm 1.0$ & $1.9 \pm 0.7$ \\
\hline AGT & $1.0 \pm 0.7$ & $0.5 \pm 0.6$ & $0.4 \pm 0.2$ & $0.7 \pm 0.6$ & $0.8 \pm 0.9$ & $1.0 \pm 1.3$ \\
\hline Rennin & $15.4 \pm 13.7$ & $18.0 \pm 11.5$ & $16.6 \pm 14.9$ & $2.4 \pm 2.8$ & $1.8 \pm 2.0$ & $0.8 \pm 0.2$ \\
\hline (P)RR & $1.0 \pm 0.2$ & $0.9 \pm 0.2$ & $1.2 \pm 0.2$ & $0.8 \pm 0.1$ & $0.7 \pm 0.0$ & $1.1 \pm 0.2$ \\
\hline TGF- $\beta$ & $1.4 \pm 1.0$ & $1.4 \pm 1.0$ & $1.0 \pm 0.2$ & $1.4 \pm 0.5$ & $0.8 \pm 0.3^{*}$ & $1.6 \pm 0.6$ \\
\hline
\end{tabular}

Abbreviations: ACE, angiotensin converting enzyme; AGT, angiotensinogen; GAPDH, glyceraldehyde-3-phosphate dehydrogenase; HRP, handle region peptide; (P)RR, (pro)renin receptor; TGF- $\beta$, transforming growth factor beta.

Data shown are expressed as mean \pm s.d.

${ }^{*} P<0.05$ vs. vehicle.

\section{DISCUSSION}

Two-kidney, one-clip Goldblatt hypertension is caused by the increased synthesis and release of renin from the clipped kidney as a result of the reduced renal perfusion pressure by unilateral stenosis of the renal artery. In this model of hypertension, decreased perfusion flow is known to be a primary pathogenic factor for acute nephropathy occurring in the clipped kidneys of $2 \mathrm{~K} 1 \mathrm{C}$ rats. In fact, renin mRNA levels increased in the clipped kidneys at both 2 and 12 weeks after clipping. Thus, the acute nephropathy occurring in the clipped kidneys is caused by ischemia and the products of renin mRNA. However, the (P)RR blocker HRP had no beneficial effect on the clipped kidneys at 2 or 12 weeks after the clipping. Because the renal expression of activated prorenin decreased in the clipped kidneys, the development of acute nephropathy in the clipped kidneys is thought to be caused by renin but not by prorenin. Although HRP is capable of blocking the binding of either renin or prorenin to the $(\mathrm{P}) \mathrm{RR},{ }^{3}$ renin is an active enzyme without $(\mathrm{P}) \mathrm{RRs}$ and is capable of generating Ang peptides, leading to the development of nephropathy. Thus, blocking (P)RR with HRP would have no effect on renin-dependent pathogenesis, as shown in previous studies. ${ }^{8,14}$

In contrast, 12-week treatment with HRP significantly attenuated the slow progression of glomerulosclerosis and TD in the non-clipped kidneys of $2 \mathrm{~K} 1 \mathrm{C}$ rats. In the early stage of renovascular hypertension, renin secretion from the non-clipped kidney is reduced by the elevated perfusion pressure. ${ }^{15}$ In contrast, the elevated pressure inhibits the processing of prorenin to renin, leading to accumulation of prorenin in renal juxtaglomerular cells, ${ }^{16}$ and the tissue Ang II levels of the nonclipped kidneys rise gradually during the late stage of renovascular hypertension, ${ }^{17}$ probably because of a prorenin-dependent mechanism. In the present study, activated prorenin and tissue Ang II levels were upregulated without any changes in (P)RR mRNA expression in the non-clipped kidneys. The long-term administration with HRP, suppressed both the elevation of activated prorenin expression and tissue Ang II levels. Thus, the increased tissue Ang II levels could be attributable to the glomerulosclerosis and the TD developed in the non-clipped kidneys with the tissue accumulated prorenin and its activation.

At 12 weeks, but not at 2 weeks, after the clipping, the treatment with HRP significantly decreased renal Ang II levels and attenuated the progression of glomerulosclerosis and TD in the non-clipped kidneys of renovascular Goldblatt hypertensive rats. Because the renal expression of activated prorenin and renal Ang II levels were not elevated at 2 weeks after clipping, HRP had no beneficial effects on either of the kidneys of the rats. These results suggest that the $(\mathrm{P}) \mathrm{RR}$ is involved in the pathogenesis of kidney damage, which slowly progresses as the renal prorenin levels increase.

In the present study, proteinuria and hypertension were not improved by the treatment with HRP, although the HRP treatment significantly inhibited the development of nephropathy in the nonclipped kidneys. This may be because of the unchanged whole kidney function, suggested by the persistent TD of the clipped kidneys and the unchanged circulating renin-angiotensin system, represented by unaffected plasma renin activity.

Elevated concentrations of TGF- $\beta$ have an important role in the pathogenesis of glomerulosclerosis and TD. ${ }^{18}$ In $2 \mathrm{~K} 1 \mathrm{C}$ renovascular hypertensive rats, at $21+$ days after clipping, TGF- $\beta$ mRNA levels have been reported to be upregulated in the non-clipped kidneys compared with sham-operated control kidneys. ${ }^{19}$ In addition, a complex and rich interaction between TGF- $\beta$ and the renin-angiotensin system has been observed in the kidneys of experimental animals with chronic kidney diseases. ${ }^{20}$ Previous studies demonstrated that Ang II stimulates the synthesis of TGF- $\beta$ in cultured rat vascular smooth muscle cells, ${ }^{21}$ rat glomerular mesangial cells ${ }^{22}$ and cultured murine proximal tubular cells. ${ }^{23}$ In addition, blocking Ang II with ACE inhibitors or 
Ang II type 1 receptor antagonists decreased the overexpression of TGF- $\beta$ in animals with chronic kidney diseases, ${ }^{20}$ and the blocking of prorenin binding to the (P)RR by HRP inhibited the elevated Ang II levels in the kidneys of the animals with diabetes ${ }^{4}$ and hypertension. ${ }^{7}$ In the present study, the treatment with HRP significantly decreased the activated prorenin levels, Ang II levels and TGF- $\beta$ mRNA levels in the non-clipped kidney. On the basis of these findings, the blocking of the $(\mathrm{P}) \mathrm{RR}$ binding to prorenin by HRP resulted in the decrease in renal levels of activated prorenin and subsequent decrease in renal Ang II levels, which led to the downregulation of TGF- $\beta$ expression and the subsequent attenuation of glomerulosclerosis and TD.

Interestingly, renal ACE mRNA levels were suppressed in the clipped kidneys, but not in the non-clipped kidneys, of the rats treated with either HRP or the scramble peptide. This result suggests non-specific effects of amino acids on ACE mRNA expression. However, neither treatment had a beneficial effect on the glomerulosclerosis and the TD occurring in the clipped kidney. Thus, the non-specific effects of amino acids seemed not to be involved in the benefits provided by HRP treatment in the nonclipped kidneys.

In conclusion, the inhibitory effects of HRP treatment on the slow progression of glomerulosclerosis and TD in the non-clipped kidneys was shown at 12 weeks, but not at 2 weeks, after the start of its administration. HRP treatment also resulted in the downregulation of activated prorenin, Ang II, and TGF- $\beta$ mRNA levels in the nonclipped kidneys, suggesting that those factors are at least partly involved in the mechanisms accounting for the beneficial effects of HRP. Thus, the (P)RR-dependent activation of prorenin contributes to the pathogenesis of the slowly progressive nephropathy (but not acute nephropathy) that developed in the intact kidneys of renovascular hypertension.

\section{ACKNOWLEDGEMENTS}

This work was supported in part by a grant from the Ministry of Education, Culture, Sports, Science, and Technology (22390171, to A. I.). We thank Chika Miki for her excellent technical assistance.

1 Nguyen G, Delarue F, Burckle C, Bouzhir L, Giller T, Sraer J-D. Pivotal role of the renin/prorenin receptor in angiotensin II production and cellular responses to renin. J Clin Inves 2002; 109: 1417-1427.

2 NurunNabi AHM, Biswas KB, Nakagawa T, Ichihara A, Inagami T, Suzuki F. 'Decoy peptide' region (RIFLKRMPSI) of prorenin prosegment plays a crucial role in prorenin binding to the (pro)renin receptor. Int J Mol Med 2009; 24: 83-89.
3 NurunNabi AHM, Biswas KB, Nakagawa T, Ichihara A, Inagami T, Suzuki F. Prorenin has high affinity multiple binding sites for (pro)renin receptor. Biochim Biophys Acta 2009; 1794: 1838-1847.

4 Ichihara A, Hayashi M, Kaneshiro Y, Suzuki F, Nakagawa T, Tada Y, Koura Y, Nishiyama A, Okada H, Uddin MN, Nabi AHMN, Ishida Y, Inagami T, Saruta T. Inhibition of diabetic nephropathy by a decoy peptide corresponding to the 'handle' region for non-proteolytic activation of prorenin. J Clin Invest 2004; 114: 1128-1135.

5 Ichihara A, Sakoda M, Mito-Kurauchi A, Nishiyama A, Itoh H. Involvement of receptorbound prorenin in development of nephropathy in $\mathrm{db} / \mathrm{db}$ mice. J Am Soc Hypertens 2008; 2: 332-340.

6 Susic D, Zhou X, Frohlich ED, Lippton H, Knight M. Cardiovascular effects of prorenin blocking blockade in genetically hypertensive rats (SHR) on normal and high salt diet. Am J Physiol Heart Circ Physiol 2008; 295: H1117-H1121.

7 Ichihara A, Kaneshiro Y, Takemitsu T, Sakoda M, Nakagawa T, Nishiyama A, Kawachi H, Shimizu F, Inagami T. Contribution of non-proteolytically activated prorenin in glomeruli to hypertensive renal damage. J Am Soc Nephrol 2006; 17: 2495-2503.

8 Muller DN, Klanke B, Feldt S, Cordasic N, Hartner A, Schmieder RE, Luft FC, Hilgers KF. (Pro)renin receptor peptide inhibitor 'handle-region' peptide does not affect hypertensive nephrosclerosis in Goldblatt rats. Hypertension 2008; 51: 676-681.

9 Satofuka S, Ichihara A, Nagai N, Noda K, Ozawa Y, Fukamizu A, Tsubota K, Itoh H, Oike $Y$, Ishida $S$. (Pro)renin receptor-mediated signal transduction and tissue renin-angiotensin system contribute to diabetes-induced retinal inflammation. Diabetes 2009; 58: 1625-1633.

10 Remuzzi G, Zoja C, Gagliardini E, Corna D, Abbate M, Benigni A. Combining an antiproteinuric approach with mycophenolate mofetil fully suppresses progressive nephropathy of experimental animals. J Am Soc Nephrol 1999; 10: 1542-1549.

11 Nishiyama A, Seth DM, Navar LG. Renal interstitial fluid angiotensin I and angiotensin II concentrations during local angiotensin-converting enzyme inhibition. J Am Soc Nephrol 2002; 13: 2207-2212.

12 Ichihara A, Kaneshiro Y, Takemitsu T, Sakoda M, Suzuki F, Nakagawa T, Nishiyama A, Inagami T, Hayashi M. Non-proteolytic activation of prorenin contributes to development of cardiac fibrosis in genetic hypertension. Hypertension 2006; 47: 894-900.

13 Bolbrinker J, Markovic S, Wehland M, Melenhorst WBWH, vanGoor H, Kreutz R. Expression and response to angiotensin-converting enzyme inhibition of matrix metalloproteases 2 and 9 in renal glomerular damage in young transgenic rats with renindependent hypertension. J Pharmacol Exp Ther 2006; 316: 8-16.

14 Feldt S, Maschke U, Dechend R, Luft FC, Muller DN. The putative (pro)renin receptor blocker HRP fails to prevent (pro)renin signaling. J Am Soc Nephrol 2008; 19: 743-748.

15 Navar LG, Zou L, VonThun A, Wang CT, Imig JD, Mitchell KD. Unraveling the mystery of Goldblatt hypertension. News Physiol Sci 1998; 13: 170-176.

16 Ichihara A, Suzuki H, Miyashita Y, Naitoh M, Hayashi M, Saruta T. Transmural pressure inhibits prorenin processing in juxtaglomerular cell. Am J Physiol 1999; 277: R220-R228.

17 Navar LG, Inscho EW, Majid SA, Imig JD, Harrison-Bernard LM, Mitchell KD. Paracrine regulation of the renal microcirculation. Physiol Rev 1996; 76: 425-536.

18 Border WA, Noble NA. Transforming growth factor beta in tissue fibrosis. N Engl J Med 1994; 331: 1286-1292.

19 Wolf G, Schneider A, Wenzel U, Helmchen U, Stahl RAK. Regulation of glomerular TGF-beta expression in the contrlateral kidney of two-kidney, one-clip hypertensive rats. J Am Soc Nephrol 1998; 9: 763-772.

20 Border WA, Noble NA. Interactions of transforming growth factor-beta and angiotensin II in renal fibrosis. Hypertension 1998; 31: 181-188.

21 Gibbons GH, Pratt RE, Dzau VJ. Vascular smooth muscle cell hypertrophy vs hyperplasia. J Clin Invest 1992; 90: 456-461.

22 Kagami S, Border WA, Miller DE, Noble NA. Angiotensin II stimulates extracellular matrix protein synthesis through induction of transforming growth factor-beta expression in rat glomerular mesangial cells. J Clin Invest 1994; 93: 2431-2437.

23 Wolf G, Mueller E, Stahl RAK, Ziyadeh FN. Angiotensin II-induced hypertrophy of cultured murine proximal tubular cells is mediated by endogenous transforming growth factor-beta. J Clin Invest 1993; 92: 1366-1372. 